\title{
Métodos e práticas colaborativas na cadeia de suprimentos: revisão de literatura
}

\author{
JoæéAlan BarbosadaSIva Especialista em Gestão de Negócios e em Gestão Estratégica de Logística. Universidade \\ Estadual de Goiás (UEG) - Brasil. is.josealan@hotmail.com
}

\section{RESUMO}

A criação das redes interorganizacionais tem por objetivo possibilitar a aplicação de métodos cooperativos que contribuam para melhorar o resultado empresarial. Diante disso, as organizações buscam maior integração, competitividade, redução de custos, maior produtividade e rentabilidade organizacional. Neste contexto, os métodos cooperativos na cadeia de suprimentos, são relevantes para tornar possível os métodos práticos de colaboração interorganizacional. 0 objetivo deste trabalho é apresentar os principais métodos colaborativos aplicados no gerenciamento da cadeia de suprimentos, buscando identificar suas características, vantagens e desvantagens, bem como, sua aplicabilidade e dificuldades de implementação no cenário brasileiro. A metodologia utilizada foi sobre o prisma de Cronin, Ryan e Coughlan (2008), aplicando revisão de literatura e contribuição teórica. Os resultados indicam contribuições de cunho gerencial e leitura que possibilitam ao leitor fácil compreensão sobre qual melhor escolha e aplicação de método cooperativo, além de divulgação e geração de conhecimento para a literatura. Identifica-se que essa prática ainda se demonstra embrionária no cenário empresarial brasileiro.

Palavras-chave: Cadeia de suprimentos. Cooperação. Colaboração interorganizacional. Eficiência logística.

\section{Methods and collaborative practices in supply chain management: literature review}

\begin{abstract}
The purpose of inter-organizational networking is to enable cooperative methods to be used to improve business performance. Therefore, organizations seek greater integration, competitiveness, cost reduction, higher productivity and organizational profitability. In this context, the cooperative methods in the supply chain are relevant to enable the practical methods of inter-organizational collaboration. In this perspective, the objective of this work is to present the main collaborative methods applied in the supply chain management, seeking to identify its characteristics, advantages and disadvantages, as well as its applicability and implementation difficulties in the Brazilian scenario. The methodology used was on the prism of Cronin, Ryan and Coughlan (2008), applying literature review and theoretical contribution. The results indicate managerial and reading contributions of easy understanding, enabling the reader to make the best choice and application of a cooperative method, as well as dissemination and generation of knowledge for literature. It is also identified that this practice is still embryonic in the Brazilian business scenario.
\end{abstract}

Keywords: Supply chain. Cooperation. Inter-organizational collaboration. Logistic efficiency. 


\section{INTRODUÇÃO}

$\mathrm{Na}$ contemporaneidade, as organizações estão inseridas em um ambiente de constantes transformações e com elevado nível de competividade. Diante disso, as organizações buscam métodos e técnicas que possam contribuir para a solução de problemas, redução de custos, aumento da satisfação dos clientes e criação de vantagens competitivas (WANKE, 2010; DA SILVA 2018). É neste cenário que as estratégias organizacionais buscam criar redes de cooperação que possam contribuir para a obtenção de vantagem competitiva e melhoria dos processos (DA SILVA; SILVA, 2018; DA SILVA, 2018; NOVAES, 2007).

Segundo Wanke (2010), a gestão da cadeia de suprimentos atualmente é considerada como uma poderosa ferramenta estratégica pelas organizações na busca por inovações, gestão eficiente do fluxo de materiais, produtos e informações, tendo por objetivo melhorar o relacionamento entre fornecedores, indústria e clientes. Além disso, o acesso à informação aumentou a consciência local e global, contribuindo para a crescente pressão dos consumidores, ativistas, organizações não governamentais (ONGs), stakeholders (partes interessadas), sobre responsabilidade ambiental e sustentabilidade, exigindo que as organizações busquem práticas que também considerem o desempenho ambiental (sustentável) e a minimização de agentes poluentes na natureza (HASSINI; SURTI; SEARCY, 2012; DA SILVA, 2018; ASRAWI; SALEH; OTHMAN, 2017).

Assim, para atingir as exigências atuais, as organizações precisam considerar que as cadeias de suprimentos são relevantes para obtenção de estratégias competitivas, em mercados que demandam cada vez mais ciclos de produtos menores, elevado nível de serviços, inovação e preços baixos (ASHBY; LEAT; HUDSON-SMITH, 2012; SIMCHI-LEVI, A; KAMINSKY; SIMCHI-LEVI, D, 2010; DA SILVA, 2018).

0 avanço tecnológico, relacionado a comunicação e transportes, conduziram as organizações ao aprimoramento contínuo da cadeia de sup rimentos e da gestão de seus processos, com a finalidade de obter vantagem competitiva, melhorando o market share (participação no mercado) (DA SILVA, 2018). Neste sentido, a estratégia da cadeia de suprimentos deve implementar parcerias e integrar seus processos com: fornecedores, produção, centros de distribuição, varejistas, atacadistas, depósitos, e clientes, buscando o gerenciamento e a integração eficiente entre todos os participantes da cadeia, de forma que o produto seja entregue no momento certo, na quantidade certa, no local certo, atendendo ao nível de serviço requisitado pelos clientes, ao mesmo tempo em que é possível minimizar os custos totais do produto final (BALLOU, 2009; CHOPRA; SODHI, 2004; CHRISTOPHER; LEE, 2004; DA SILVA; SILVA, 2018; DA SILVA; SCHLAG, 2017; MENTZER et al., 2001; DA SILVA, 2018).

Nesta lógica, o nível de serviço ofertado ao cliente, dependerá do esforço conjunto de todos os integrantes da cadeia de sup rimentos (NOVAES, 2007; DA SILVA, 2018). Nesta visão, as estratégias que buscam obter vantagens competitivas, melhorando a eficiência, eficácia e efetividade dos processos e operações, transcendem o âmbito intraorganizacional para o interorganizacional, ou seja, a vantagem competitiva atual se dá no âmbito cooperativo entre as cadeias produtivas e de suprimentos (GOMES, 2014; DA SILVA, 2018).

Dessa forma, para que o gerenciamento da cadeia de suprimentos seja eficiente e eficaz, e possa melhorar a competitividade, é necessário cooperação, integração, compartilhamento de informações, e, principalmente, esforço conjunto entre todos os participantes da cadeia de suprimentos da organização (PIRES, 2007; DA SILVA, 2018). Os métodos e práticas colaborativas que se destacam são: o Supply Chain Operations Reference-model (SCOR); o Quick Response (QR); o Continuos Replenishment (CR); o Customer Service Logistics (CSL); o Vendor Management Inventory (VMI); o Efficient Consumer Response (ECR); e o Collaborative Planning Forecasting Replenishment (CPFR) (DA SILVA, 2018; WANKE, 2010; YAOA, et al. 2013).

A fim de demonstrar o cenário atual, através da contribuição teórica em revisão da literatura, so bre os métodos e práticas relacionadas ao gerenciamento da cadeia de suprimentos, este artigo tem como objetivo contribuir com a discussão de possibilidades metodológicas para estudos organizacionais dentro da abordagem das técnicas colaborativas utilizadas na Gestão da Cadeia de Suprimentos (GCS) ou Supply Chain Management (SCM), buscando analisar e investigar o empenho de outros estudos com vistas a ampliar as possibilidades teóricas e empíricas. 
Esse trabalho se justifica pelo cenário atual em que as organizações estão inseridas, e pela necessidade de trabalhos e pesquisas que abordem na produção acadêmica brasileira, especialmente, a discussão acerca das possibilidades e perspectivas que caracterizam a Supply Chain Management ou Gestão da Cadeia de Suprimentos e aponte práticas que favoreçam o desenvolvimento das empresas (DA SILVA, 2018; WANKE, 2010; PIRES, 2007; GOMES; KLIEMANN NETO, 2015; BALLOU, 2009; ABDALA; BARBIERI, 2014; DA SILVA; SILVA, 2018; DA SILVA; SCHLAG, 2017; MENTZER et al., 2001).

Este artigo está constituído com as seguintes seções: 1 (um) introdução, apresentando relevância, objetivo e resultado esperado deste estudo, a seção 2 (dois) traz a base teórica que fundamenta o trabalho; a seção 3 (três) apresenta o procedimento metodológico; a seção 4 (quatro) apresenta a avaliação e os resultados obtidos; e, finalmente, a seção 5 (cinco) que apresenta as considerações finais sobre esta pesquisa e sugestões para futuros trabalhos.

\section{PLATAFORMA TEÓRICA}

De acordo com Calatayud, Mangan e Christopher (2019), a cadeia de sup rimentos, é formada por uma rede de organizações com processos cooperativos a jusante (downstream) e a montante (upstream), nas diferentes operações e processos, que tem por objetivo criar e agregar valor na forma de produto e níveis de serviços aos clientes e aos consumidores finais.

O cenário econômico atual é caracterizado por um mercado intensamente globalizado, em que as empresas buscam cada vez mais estratégias e vantagens competitivas, através da cooperação interorganizacional entre as cadeias de suprimentos da indústria e do agronegócio, buscando integração das atividades, maior colaboração e desenvolvimento tecnológico, maior oferta de produtos, menores custos operacionais e ampliação dos serviços aos clientes (VERSCHOORE; BALESTRIN, 2008; ABDALLAH; BARBIERI, 2014; DA SILVA, 2018; GOMES; KLIEMANN NETO, 2015).

Neste contexto, a ampliação das relações empresariais, fez com que muitas empresas procurassem estabelecer redes de cooperação entre indústria, produtores de matéria-prima, fornecedores de peças e componentes, distribuidores e varejistas, que são os agentes que fazem parte da cadeia produtiva e de suprimentos, buscando melhorar o relacionamento e o resultado empresarial conjunto (CHEN, 2013; RAMANATHAN; GUNASEKARAN, 2014; DA SILVA, 2018).

Com isto, a gestão colaborativa na cadeia de suprimentos, passou a representar estratégia fundamental na busca por ganhos de escala, aumento da capacidade em atender diferentes mercados e segmentos (do produtor de matéria-prima, passando pelo processo de produção até a entrega do produto ao cliente final) tornando a cadeia mais ágil e com maior flexibilidade, com melhores níveis de serviços aos clientes e com menores custos de operação (ELLRAM; COOPER, 2014; DA SILVA, 2018).

Nesta lógica, a amplitude de gestão da cadeia de suprimentos envolve redes e colaboração interorganizacional, melhoria no relacionamento entre empresas, compartilhamento de riscos e objetivos comuns. Esse esforço conjunto, na prática, é responsável por determinar o quão eficiente é a cadeia produtiva e quais serão os custos incorridos nas operações logísticas e do transporte, até disponibilizar o produto para o consumidor final (BALESTRIM; VERSCHOORE, 2014; CAO; ZHANG, 2011; DA SILVA, 2018; RAMANATHAN, 2012; LIU et al., 2013).

Assim, a gestão cooperativa na cadeia de suprimentos das empresas, surge como necessidade no cenário atual, contribuindo para a implementação de projetos que colaboram com a sociedade, ao possibilitar a oferta de produtos com maior qualidade, melhores níveis de serviços e menores custo de aquisição (BALLOU, 2009; DA SILVA; SILVA, 2018; DA SILVA, 2018; HUDNURKAR; JAKHAR; RATHOD, 2014).

Em síntese, podemos afirmar que a gestão da cadeia de suprimentos, surge como um novo modelo estratégico gerencial, que busca alcançar sinergia e integração dos processos-chave do negócio ao longo de uma cadeia produtiva (PIRES, 2009; VAN HOEK; CHAPMAN, 2007; DA SILVA, 2018). 


\subsection{Colaboração e Métodos Cooperativos na Gestão da Cadeia de Suprimentos}

Conforme enfatizado por Pires (2009), e, Da Silva, (2018), quando as organizações gerenciam de forma estratégica a cadeia de suprimentos é possível desenvolver processos que permitem uma gestão mais eficiente, precisa e objetiva, como consequência os processos, operações e atividades são melhores desempenhadas.

Diante disso, iremos apresentar sete métodos cooperativos mais discutidos e conhecidos na literatura: o Supply Chain Operations Reference-model (SCOR); o Quick Response (QR); o Continuos Replenishment Program (CRP); o Customer Service Logistics (CSL); o Vendor Management Inventory (VMI); 0 Efficient Consumer Response (ECR); e o Collaborative Planning Forecasting Replenishment (CPFR).

Uma das técnicas mais utilizadas é o modelo de referência para as operações da cadeia de suprimentos, do inglês Supply Chain Operations Reference-model (SCOR), proposto pelo Conselho de Profissionais de Gestão da Cadeia de Suprimentos, ou do inglês Council of Supply Chain Management Professionals (CSCMP, 2018).

De acordo com o CSCMP (2018), o modelo de referência para as operações da cadeia de suprimentos (SCOR), é reconhecido como sendo o primeiro modelo de referência construído para descrever, comunicar, avaliar e melhorar o desenho da cadeia de suprimentos, ou seja, sua essência é orientada para a tomada de decisões. Além disso, esse modelo possibilita a integração da cadeia de suprimentos, ao definir seus processos de negócios através de indicadores que ajudam a monitorar, controlar e mensurar os processos e operações logísticas (DA SILVA, 2018). Este modelo possui cinco processos: Plan (Planejar); Source (Abastecer); Make (Fazer); Deliver(Entregar); e, Return (Retornar).

Dando sequência, o segundo método apresentado, e conforme enfatizado por Wanke (2010), o Quick Response (QR) ou Resposta Rápida, é uma técnica responsável pelo intercâmbio de informações desde os pontos de venda até os fornecedores, onde ocorre o compartilhamento de informações em tempo real, entre os participantes da cadeia, possibilitando o planejamento em relação à oferta x demanda.

O terceiro método é apresentado por Bertaglia (2003), conhecido como Efficient Consumer Response (ECR) ou Resposta Eficiente ao Consumidor, possui como foco principal, compreender quais são as necessidades do consumidor, objetivando criar e agregar valor no produto e na adequação da prestação de serviço para o cliente, em todas as esferas da cadeia de suprimentos.

Para Guarnieri, et al. (2006), Ching (2010), e, Da Silva (2018), a agregação de valor para o cliente somente ocorre quando o próprio cliente percebe a melhoria na qualidade dos produtos, dos preços e da qualidade da entrega, bem como a diversidade de produtos para a sua escolha, ou seja, a criação de valor deve ocorrer de tal forma que seja perceptível para o cliente. Portanto, para isso é necessário que a organização desenvolva técnicas que possibilitem a redução dos níveis de estoques, dos custos e das perdas de produtos não consumidos, gerando maior eficiência e eficácia na gestão da cadeia de suprimentos. Desse modo, Ching (2010), e, Ghisi e Silva (2005), destacam que as premissas básicas para a implementação do ECR são: entrada de novos produtos; sortimento; promoção; e reposição. Esses são quatro fatores que o ECR deve acompanhar para que as operações tenham sucesso.

o Continuous Replenishment Program (CRP) ou Programa de Reabastecimento Contínuo, é o quarto método, e tem por objetivo demonstrar a necessidade do compartilhamento de informações do ponto de venda até os fornecedores, possibilitan do manter, controlar e coordenar a gestão de estoques (níveis mínimos e máximos) (WANKE, 2010; DA SILVA, 2018). Com a implementação dessa técnica, pretende-se utilizar de forma efetiva as informações que são geradas e obtidas no cliente, para ajustar os lotes de produção dos fornecedores e da própria indústria de transformação (DA SILVA, 2018; MATHIS, et al. 2005; NAGALATHA; HUSSAIN, 2011).

O quinto método, conhecido como VendorManagement Inventory(VMI) ou Estoque Gerenciado pelo Fornecedor, é um método utilizado para melhorar o gerenciamento dos estoques de seus clientes, onde a gestão fica sob responsabilidade do fornecedor, que passa a analisar a previsão de demanda, programando suas atividades de forma conjunta, tornando efetivo o processo de produção, transporte e distribuição, 
minimizando os custos com gargalos, movimentações e estoques desnecessários (WANKE, 2010; CORRÊA, H; CORREAA, C, 2007; PISSAIA; CHAVES; CARDOSO, 2012; DA SILVA, 2018).

O sexto método é o Collaborative Planning Forecasting Replenishment (CPFR), ou Planejamento Colaborativo de Previsão de Reabastecimento, apesar de ser uma prática bastante utilizada pelas grandes empresas, pode ser considerado como uma inovação e/ou uma evolução dos métodos cooperativos anteriores (WANKE, 2010; DA SILVA, 2018; YAOA, et al, 2013). Na visão de Borges (2011), e Da Silva (2018), esse método tem por objetivo sanar as falhas encontradas em métodos como ECR, VMI e CR. Nesta técnica o compartilhamento de informações ocorre por meio de sistemas e são analisadas as previsões de demandas. Uma das vantagens desse método é tornar possível analisar e identificar em toda a cadeia de suprimentos, quais são os integrantes que possuem melhor previsão de demanda quanto as vendas de um Stock Keeping Unit (SKU) ou Unidade de Controle de Estoque, levando em consideração o lead time (tempo) e a localização da demanda (WANKE, 2010; DA SILVA, 2018).

O sétimo método é o Customer Service Logistics (CSL) ou Logística de Atendimento do Cliente, ou ainda, Serviço de Logística ao Cliente, que tem como premissa, atuar em conjunto com a Gestão da Cadeia de Suprimentos, como uma ferramenta importante na busca do atendimento e melhoria do serviço logístico ao cliente (DA SILVA, 2018). 0 principal objetivo dessa técnica é a construção de projetos que adequam esforços na direção correta das necessidades dos clientes, com isso, é possível reduzir custos operacionais, tanto para o fornecedor, quanto para os clientes e consequentemente o preço dos produtos para o consumidor final (DA SILVA, 2018; DAVIS; MENTZER, 2011; TONTINI; ZANCHETT, 2010; BANOMYONG; SUPATN, 2011).

Para Salzano (2008), e, Da Silva, (2018), a função do Customer Service Logisticsé analisar, implementar, gerenciar e mensurar o melhor nível de serviço, ou o mais apropriado, considerando as necessidades, exigências e expectativa dos clientes.

\section{PROCEDIMENTOS METOLÓGICOS}

Uma pesquisa precisa atingir um nível adequado de confiabilidade e sucesso a partir da definição de processo e metodologias sistemáticas, instrumentos adequados e coerentes com o seu propósito, além de rigor técnico e científico para a sua aceitação (GIL, 2009; YIN, 2015; MORESI, 2003).

Diante disso, este trabalho pode ser definido como uma sistematização e contribuição teórica, da investigação de um problema a fim de se obter considerações e ou conclusões sobre determinado assunto 0 qual não possui clareza sobre seus fundamentos ou características devido à falta de organização de conhecimento e fatos.

Nesta lógica, realizou-se uma revisão sistemática de literatura, que sobre o prisma de Cronin, Ryan e Coughlan (2008), a contribuição teórica e revisão de literatura é a busca de trabalhos e estudos, tendo os critérios claros e bem definidos para que seja possível atingir o objetivo proposto.

Além disso, buscou-se a realização de uma síntese sobre os métodos utilizados na cadeia de suprimentos, para isso realizou-se uma pesquisa com dados secundários que tratam e abordam a temática. Assim, emprega-se uma revisão de literatura, a metodologia permite a reprodução da pesquisa, e dessa forma, poderá contribuir com 0 entendimento do tema e até facilitar pesquisas posteriores sobre a temática (SAMPAIO; MANCINI, 2007).

Aditivamente, este trabalho analisou e revisou os estudos de caso encontrados na literatura, com o intuito de compreender quais são os métodos cooperativos mais utilizados pelas organizações, assim como, verificar quais são as vantagens e desvantagens para as empresas, constatando sua aplicabilidade no cenário empresarial. Referente aos métod os colaborativos, foram determinados os que mais se destacam na literatura. A seguir apresentam-se as seções dos registros identificados na revisão de literatura (Tabela 1). 
Tabela 1 - Seções de registros da revisão de literatura

\begin{tabular}{ll}
\hline \multicolumn{1}{c}{ Etapas da Revisão } & \multicolumn{1}{c}{ Aplicação dos Registros } \\
\hline & $\begin{array}{l}\text { Os conceitos apresentados pela literatura quanto aos sete métodos } \\
\text { colaborativos, deste trabalho, foram realmente aplicáveis em seus estudos } \\
\text { de caso? Existe consenso entre as definições dos métodos cooperativos nos } \\
\text { trabalhos pesquisados que clarificam a diferença entre eles? } \\
\text { l. Formulação da questão }\end{array}$ \\
& Quais vantagens e desvantagens dos métodos? \\
\hline
\end{tabular}

a) Banco de dados da busca: Google acadêmico; Periódicos da Science Direct,

b) Período de publicação: 2000-2018; II. Estabelecimento dos Response (QR); Continuous Replenishment Program (CRP); Vendor críterios de inclusãol e Management Inventory (VMI); Efficient Consumer Response (ECR); exclusão Collaborative Planning Forescating Replenishment (CPFR); Supply Chain Operations Reference-model; Método colaborativo; Método Cooperativo; Collaborative methods; Cooperative methods. As palavras devem aparecer no título do artigo;

d) Tipos de trabalhos: Artigos de periódicos científicos; teses e dissertações, eventos e congressos da área;

e) Citações e patentes foram excluídas.

III. Acesso e seleção à literatura Banco de dados: Google acadêmico e Science Direct.

IV. Avaliação da qualidade da literatura considerada e/ou incluída neste trabalho a) Para cada método pesquisado, o número total de artigos encontrados, foram: (i) Google Acadêmico: 135 trabalhos: QR (21); ECR (32); VMI (25); CPFR (13); SCOR (7); e, CSL (10);

b) Foram encontrados 14 artigos dentro do critério selecionado, no Portal de periódicos da Google Acadêmico: QR (3); CRP (0); ECR (2); VMI (4); CPFR (4); $\operatorname{SCOR}(1) ; \mathrm{e}, \mathrm{CSL}(1)$;

c) Foram encontrados dois trabalhos no Science Direct. VMI (1); e CPFR (1).

Foram considerados somente os artigos que especificamente eram sobre os métodos colaborativos ou cooperativos, excluindo-se citações e patentes, V. Análise, síntese e sendo incluídos estudos de casos. Além disso, considerou-se somente os disseminação dos resultados artigos que apresentaram alguma contribuição para o estudo do método em questão. Considerou-se artigos em português, espanhol e inglês. Artigos sem referência explícita também foram excluídos.

Fonte: Autor (2018).

Por fim, o material coletado foi analisado, comparado e discutido sistematicamente, sendo que dos 717 artigos encontrados, apenas 17 artigos atingiram os critérios da pesquisa. Os resultados e discussão são apresentados no próximo tópico. 


\section{RESULTADOS E DISCUSSÃO}

Esta seção, apresenta os resultados, análises e discussões dos resultados. 0 material identificado através da revisão de literatura, de acordo com a seção de registros de Cronin, Ryan e Coughlan (2008), conforme demonstrado no Quadro 1.

Quadro 1 - Resultados da revisão de literatura sobre métodos colaborativos

\begin{tabular}{|c|c|c|c|c|}
\hline Base & Método & Autor (es) & Título do artigo & Dados dos artigos \\
\hline \multirow{7}{*}{ 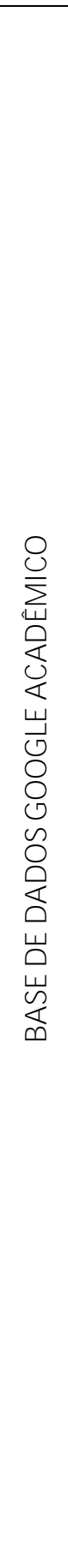 } & 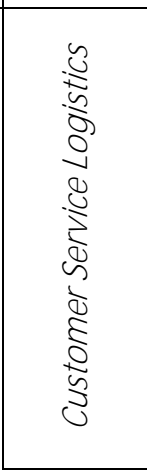 & DA SILVA (2018). & $\begin{array}{l}\text { Customer Service } \\
\text { Logistics como } \\
\text { Estratégia } \\
\text { Organizacional: um } \\
\text { estudo de caso em } \\
\text { uma multinacional. }\end{array}$ & $\begin{array}{l}\text { Estudo sobre a aplicação das técnicas } \\
\text { e ferramentas do Customer Service } \\
\text { Logistics como estratégia } \\
\text { organizacional. Estudo de caso } \\
\text { aplicado em uma multinacional com } \\
\text { atuação global. Concluiu-se que as } \\
\text { técnicas do CSL contribuíram para a } \\
\text { experiência de compra positiva do } \\
\text { cliente, melhoria dos níveis de serviços } \\
\text { logísticos, além de fidelização e } \\
\text { manutenção dos clientes/vendas. }\end{array}$ \\
\hline & \multirow{3}{*}{ 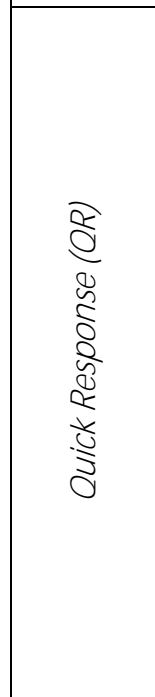 } & $\begin{array}{c}\text { D. MAO, D. H.; } \\
\text { ZHU, Y. M. CU. (2011). }\end{array}$ & 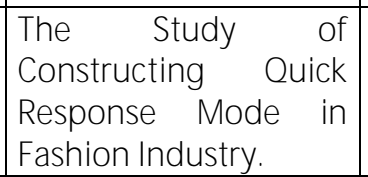 & $\begin{array}{l}\text { Estudo sobre a relação entre o Quick } \\
\text { Response (QR) e a indústria da mora. } \\
\text { Estudo de caso realizado na empresa } \\
\text { Zara. }\end{array}$ \\
\hline & & ZHANG, C. J. (2012). & $\begin{array}{lrr}\text { Study } & \text { on } & \text { Quick } \\
\text { Response } & \text { (QR) } & \text { Mode } \\
\text { of } & \text { Agricultural } \\
\text { Products } & \text { Logistics } \\
\text { under } & \text { E-commerce } \\
\text { Environment. }\end{array}$ & $\begin{array}{l}\text { Análise da conotação do Quick } \\
\text { Response (QR)e de suas atribuições na } \\
\text { logística. Estudo de caso sobre a APEL } \\
\text { (Agricultural Products E-comerce } \\
\text { Logistics) na província de Hubei da } \\
\text { cidade de Wuhan (China). }\end{array}$ \\
\hline & & YAN, S. (2013). & $\begin{array}{l}\text { Empirical study on } \\
\text { influencing factors of } \\
\text { Quick Response (QR) } \\
\text { ability for small and } \\
\text { medium sized logistics } \\
\text { enterprise. }\end{array}$ & $\begin{array}{l}\text { Estudo empírico, com aplicações de } \\
\text { questionários em } 500 \text { organizações de } \\
\text { pequeno e médio porte, referente aos } \\
\text { fatores de influência do } Q R \text {, com base } \\
\text { no software AMOS } 7 \text {. }\end{array}$ \\
\hline & جُ̂ & $\begin{array}{c}\text { Não foram encontrados } \\
\text { artigos. }\end{array}$ & & \\
\hline & \multirow[t]{2}{*}{$\bar{\xi}$} & $\begin{array}{c}\text { GUIMARAES, L. G; } \\
\text { MACIEL, T, K; } \\
\text { GARCIA, V. J. (2014). }\end{array}$ & $\begin{array}{l}\text { Case } \\
\text { Implementation of } \quad \text { of } \\
\text { Vendor Managed } \\
\text { Inventory (VMI) in a } \\
\text { Company to Slaughter } \\
\text { Large Birds. }\end{array}$ & $\begin{array}{l}\text { Estudo qualitativo realizado para } \\
\text { atestar se há viabilidade de } \\
\text { implementar o Vendor Managed } \\
\text { Inventory (VMI)em um abatedouro de } \\
\text { aves. O estudo de caso foi aplicado no } \\
\text { estado do Rio Grande do Sul para } \\
\text { verificar a gestão de estoques. } \\
\text { Concluiu-se que a implementação é } \\
\text { viável }\end{array}$ \\
\hline & & HASSAIN, M. N. (2011). & $\begin{array}{lrr}\text { Potencial } & \text { of } & V M I \\
\text { aplication } & \text { in } \\
\text { Commercial } & \text { Vehicle } \\
\text { Manufacturing } & \\
\text { Industry: } \text { A Case Study. }\end{array}$ & $\begin{array}{l}\text { Estudo de caso em uma fábrica de } \\
\text { veículos comerciais chamada ASHOK } \\
\text { LEYLAND (AL) e localizada na Índia. }\end{array}$ \\
\hline
\end{tabular}




\begin{tabular}{|c|c|c|c|}
\hline & $\begin{array}{l}\text { LUO, R.; HUANG, J.; } \\
\text { LEE, J.; PUN, P. (2017) }\end{array}$ & $\begin{array}{l}\text { Strategic Alliances in } \\
\text { Notebook PC Supply } \\
\text { Chain: A Case Study A } \\
\text { Hard Disk Drive } \\
\text { Supplier } \\
\text { Implementing VMI in } \\
\text { China. }\end{array}$ & $\begin{array}{l}\text { Estudo de caso sobre uma empresa } \\
\text { japonesa de notebooks que se mudou } \\
\text { para a China. Identificado que a } \\
\text { empresa tinha problemas com a falta } \\
\text { de produto no estoque para atender } \\
\text { clientes e demora na entrega de } \\
\text { cargas dos navios. O estudo é voltado } \\
\text { para a compreensão de como esses } \\
\text { problemas foram solucionados por } \\
\text { meio das alianças estratégicas } \\
\text { implementadas. }\end{array}$ \\
\hline & SUN, Y. (2013). & $\begin{array}{l}\text { Study on } \\
\text { Manufacturing } \\
\text { Industry Based on VMI. }\end{array}$ & $\begin{array}{l}\text { Estudo de caso realizado em uma } \\
\text { fábrica na China com produtos } \\
\text { elétricos para trabalho, jardinagem e } \\
\text { outros. A organização possui mais de } \\
100 \text { integrantes em sua Supply Chain } \\
\text { Management. }\end{array}$ \\
\hline & $\begin{array}{l}\text { ZVIRGZDINA, R.; LININA, } \\
\text { VEVERE, V. (2015). }\end{array}$ & $\begin{array}{lr}\text { Efficiente } & \text { Consumer } \\
\text { Response (ECR) } \\
\text { Principles and theis } \\
\text { Application in Retail. } \\
\end{array}$ & $\begin{array}{l}\text { Estudo do ECR aplicado no setor de } \\
\text { varejo da Letônia. }\end{array}$ \\
\hline 䍃 & J ANETH, L. (2015). & $\begin{array}{lr}\text { La ECR (Efficient } \\
\text { Consumer Response) y } \\
\text { la Distribución de los } \\
\text { productos de la la } \\
\text { empresa DAVIAGRO } \\
\text { de la ciudad de } \\
\text { Ambato. }\end{array}$ & $\begin{array}{l}\text { Estudo de caso que analisa como o } \\
\text { ERC pode influenciar uma empresa de } \\
\text { produtos agrícola, a DAVIAGRO, } \\
\text { quanto à distribuição de produtos na } \\
\text { região. }\end{array}$ \\
\hline \multirow{4}{*}{ 畄 } & DANESE, P. (2007). & $\begin{array}{ll}\text { Designing } & \text { CPFR } \\
\text { collaborations: } & \\
\text { insights from } & \text { seven } \\
\text { case studies. } & \end{array}$ & $\begin{array}{l}\text { Apresentação de } 7 \text { casos relacionados } \\
\text { à implementação do CPFR. O artigo } \\
\text { tem por objetivo compreender o } \\
\text { processo de escolha que levou } \\
\text { empresas a optarem pelos tipos de } \\
\text { CPFR. }\end{array}$ \\
\hline & $\begin{array}{c}\text { THOMASSEN, K. M.; } \\
\text { DREYER, H.; } \\
\text { JONSSON, P. (2012). }\end{array}$ & $\begin{array}{l}\text { Exploring the Impact } \\
\text { of ICT in CPFR: A Case } \\
\text { Study of an APS } \\
\text { System in a Norwegian } \\
\text { Pharmacy Supply } \\
\text { Chain. }\end{array}$ & $\begin{array}{l}\text { Estudo sobre os impactos da utilização } \\
\text { de um sistema ICT no CPFR. O estudo } \\
\text { de caso foi aplicado em duas empresas } \\
\text { de uma cadeia de suprimentos de } \\
\text { farmácias na Noruega que se utiliza do } \\
\text { CPFR para colaboração. }\end{array}$ \\
\hline & $\begin{array}{c}\text { ZHANG, J.; } \\
\text { CHEN, W. L. (2013). }\end{array}$ & $\begin{array}{l}\text { The Study of Supply } \\
\text { Chain's Symbiotic } \\
\text { Relationship Based on } \\
\text { CPFR - In Case of Food } \\
\text { Industry. }\end{array}$ & $\begin{array}{l}\text { O artigo apresenta questões teóricas } \\
\text { sobre o CPFR e utilização de um } \\
\text { estudo de caso nas empresas Nestlé e } \\
\text { Carrefour em Taiwan. }\end{array}$ \\
\hline & $\begin{array}{c}\text { LENKA, B.; } \\
\text { BARBORA, R. (2015). }\end{array}$ & $\begin{array}{l}\text { Possibilities of } \\
\text { Deepening } \\
\text { Cooperation with } \\
\text { Suppliers Based on the } \\
\text { CPFR Method in a } \\
\text { Company Producing } \\
\text { and Supplying Fish } \\
\text { and Fish Products - } \\
\text { Case study. }\end{array}$ & $\begin{array}{l}\text { Estudo com metodologia qualitativa } \\
\text { que analisa a colaboração entre } \\
\text { fornecedores euma empresa Checa de } \\
\text { peixe de água salgada e de água doce. }\end{array}$ \\
\hline$\stackrel{r}{0}$ & $\begin{array}{c}\text { Não foram encontrado } \\
\text { artigos. }\end{array}$ & & \\
\hline
\end{tabular}




\begin{tabular}{|c|c|c|c|}
\hline 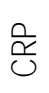 & $\begin{array}{c}\text { Não foram encontrados } \\
\text { artigos. }\end{array}$ & & \\
\hline $\bar{\xi}$ & CHEN, L. (2013). & $\begin{array}{l}\text { Dynamic supply chain } \\
\text { coordination under } \\
\text { consignment and } \\
\text { vendor - managed } \\
\text { inventory in retailer- } \\
\text { centric B2B electronic } \\
\text { markets. }\end{array}$ & $\begin{array}{l}\text { Estudo sobre a dinâmica da Supply } \\
\text { Chain Management com base em } \\
\text { consignação e contratos de VMI, com } \\
0 \text { objetivo de maximizar o lucro. } \\
\text { Estudo de caso em uma empresa } \\
\text { regional de frutos do mar com estas } \\
\text { características. }\end{array}$ \\
\hline 䍃 & \multicolumn{3}{|c|}{ Não foram encontrados artigos. } \\
\hline 䋨 & $\begin{array}{l}\text { YAOA, Y.; KOHLIN, R.; } \\
\text { SHERERA, S. A.; } \\
\text { CEDERLUNDC, J . (2013) }\end{array}$ & $\begin{array}{l}\text { Learning curves in } \\
\text { collaborative } \\
\text { planning, forecasting, } \\
\text { and replenishment } \\
\text { (CPFR) information } \\
\text { systems: An empirical } \\
\text { analysis from a Mobile } \\
\text { phone manufacturer. }\end{array}$ & $\begin{array}{l}\text { Estudo sobre as curvas de aprendizado } \\
\text { no CPFR com apresentação do estudo } \\
\text { de caso em uma empresa da Motorola } \\
\text { e um varejista. }\end{array}$ \\
\hline
\end{tabular}

Fonte: Autor (2018).

Os dados obtidos no Quadro 1, mostram que a maio ria dos artigos são internacionais e os estudos de casos foram aplicados em organizações dos setores automobilístico, cosméticos e perfumaria, moda, produção e indústria de alimentos. Esses dados demonstram uma maior preocupação destes segmentos na busca em utilizar métodos colaborativos ou cooperativos que contribuam para melhoria dos processos.

Wanke (2010) e Da Silva (2018), relatam que os motivos para estes setores buscarem os métodos são: a alta competitividade dos mercados a nível global; exigência dos consumidores por respostas rápidas as suas necessidades, principalmente no segmento da indústria de vestuário e moda, onde as coleções mudam em um curto período de tempo; é o caso da indústria de alimentos, em que a conservação dos alimentos é imprescindível, fazendo com que tal segmento necessite chegar rapidamente ao consumidor em um curto espaço de tempo. Neste contexto, os trabalhos "Case Study Implementation of Vendor Managed Inventory (VMI) in a Company to Slaughter Large Birds", e, "Customer Service Logistics como Estratégia Organizacional: um estudo de caso em uma multinacional", são de autoria brasileira. Ao considerar-se que a maioria dos estudos de caso são desenvolvidos na China, Taiwan e Europa, trabalhos como estes contribuem para ampliar e divulgar o conhecimento sobre as práticas colaborativas na gestão da cadeia de sup rimentos no Brasil.

Com exceção para o trabalho "Customer Service Logistics como Estratégia Organizacional: um estudo de caso em uma multinacional", que apresenta quantitativamente as técnicas e métodos para mensurar e gerenciar os vários processos da cadeia de suprimentos e do ciclo do pedido dos clientes, os demais artigos identificados na base de dados do Google Acadêmico, demonstram que os conceitos sobre os métodos, as vantagens e desvantagens são de certa forma generalistas quanto a delimitações. Identificou-se conceitos gerais como: redução de custos; maior controle de estoques; melhora no nível de serviço; e, maior geração de valor, porém, nenhum dos trabalhos abordou análises tangíveis e suficientemente comparativas entre outros artigos encontrados na literatura. Consequentemente, isso afeta a análise individual de cada método e a possibilidade de as organizações fazerem a melhor escolha, além de impactar nas oportunidades de futuras pesquisas sobre a temática.

Diante disso, tendo como base o material identificado, verifica-se que muitos conceitos além de serem generalistas, apresentam-se como aplicáveis a qualquer método colaborativo. Esse fato traz maior dificuldade para o entendimento dos métodos e ap licação correta das práticas de gestão cooperativa. No que diz respeito às diferenciações dos trabalhos pesquisados, as principais estão em função do setor do mercado em que o 
método foi aplicado ou mais utilizado. A exemplo disso temos o VMI que tende a ser aplicado em setores da indústria automobilística e o QR em indústrias do setor vestuário.

Referente às características mais comuns e generalistas dos métodos apresentados, temos as seguintes evidências: (a) ferramenta ou política de colaboração entre dois membros da cadeia para desenvolver relacionamentos cooperativos; (b) gerenciamento de estoques e custos; (c) utilização de sistemas e softwares como Electronic Data Interchange (EDI) ou Intercâmbio Eletrônico de Dados para troca de informações; (d) previsão de demanda a partir da integração dos dados; (e) planejamento conjunto; e (f) planejamento para otimização da produção e logística. Portanto, torna-se difícil avaliar quais as reais diferenças entre eles quando se parte de informações generalistas.

Quanto às vantagens dos métodos, as mesmas considerações generalistas foram identificadas: (a) diminuição de custos logísticos e de produção e estoques; (b) maior giro de estoques e diminuição destes; (c) eficiência na produção e no gerenciamento de estoques; (d) otimização dos lucros da cadeia; (e) maior nível de serviço; (f) capacidade de adaptação às demandas; ( $g$ ) diminuição do "efeito chicote" na cadeia de suprimentos por meio de melhores previsões de demanda; (h) planejamento mais eficiente de produção; (i) aumento da vantagens competitivas; (j) trabalho mais cooperativo entre os integrantes da cadeia; e (I) maior eficácia na comunicação da cadeia.

Já as desvantagens discutidas nos artigos levantados são praticamente nulas as quantidades de trabalhos que não as mencionam, sendo representados por $69 \%$ dos trabalhos. Quando esses métodos colaborativos não apresentam diferenças significativas em seus conceitos e vantagens, bem como, não apontam desvantagens em artigos e periódicos, é possível verificar que: (a) a utilização desses métodos é recente e ainda são desconhecidas suas reais implicações na prática; (b) a literatura não tem contribuído de forma mais direta com a utilização dos métodos para sua aplicação em empresas, pois as características apresentadas são generalistas e podem causar maior incompreensão desses métodos; (c) a recente utilização traz maiores dificuldades para as definições reais de cada método; $e$, (d) mesmo em setores consolidados com relação ao uso de métodos colaborativos, como a indústria automobilística e de vestuário ainda existem dúvidas quanto aos seus reais ganhos e desvantagens.

Assim, ao analisar os dados e material levantados, percebe-se que mesmo com conceitos mais generalistas, as análises dos artigos trazem contribuições mais específicas quanto aos métodos e suas implicações, mas ainda não são suficientes.

Os estudos de casos tem apresentado ganhos através dessa performance colaborativa, sendo que se pode encontrar melhorias, tangíveis e numéricas nos artigos: "Possibilities of Deepening Cooperation with Suppliers Based on the CPFR Method in a Company Producing and Supplying Fish and Fish Products: Case study"; "The Study of Supply Chain's Symbiotic Relationship Based on CPFR: In Case of Food Industry", e, "Customer Service Logistics como Estratégia Organizacional: um estudo de caso em uma multinacional". Percebe-se que é importante a utilização de parâmetros quantitativos e/ou numéricos para comparações entre estudos de casos na literatura.

Por fim, com base na análise do material levantado e no objetivo desse artigo,

percebe-se:

1) Alguns estudos de casos eram de conteúdo empírico, sem muitas informações concretas quanto a sua aplicação e nem sobre quais medidas foram realizadas para alavancar os resultados da empresa. Outros se configuraram em planos de propostas de aplicação ou modelos de um método colaborativo, não apresentando reais contribuições para vantagens e desvantagens. Sendo assim, seria interessante um maior nível de estudos com base nos desafios reais das organizações, para a aplicação dos métodos durante e após sua implementação, considerando-se também, mais de um fornecedor na cadeia, abrangendo-se a complexidade da implementação dos métodos;

2) Outro ponto analisado é a qualidade dos artigos referentes a esses temas. Alguns já começavam a realizar análises sobre os métodos não se utilizando de revisões de literaturas. Isso também é comprovado pelo método de eliminação de artigos usados no começo deste trabalho, em que se precisaram eliminar trabalhos que não apresentavam bibliografia; 
3) Encontrou-se também, artigos pautados apenas na utilização de sistemas de informação e não para a gestão da colaboração por meio do método. Assim, fica evidente que a apresentação dos métodos colaborativos em trabalhos é muitas vezes limitada ao uso de sistemas e trocas de informações, não abrangendo seu potencial maior de cooperação, como por exemplo, em assuntos como a colaboração na promoção de inovação e lançamento de produtos no mercado.

4) Mais uma comprovação da dificuldade de definição dos conceitos dos métodos colaborativos é apresentada pela diversidade de índices para avaliação dos ganhos dos métodos colaborativos. Isso fica evidente quanto ao método colaborativo QR no artigo "Empirical study on influencing factors of Quick Response ability for small and medium sized logistics enterprise" do Autor Shen Yan (2013). Outra evidência sobre isso é a utilização de diversos modelos de CPFR nas empresas como apresentado no artigo "Designing CPFR collaborations: insights from seven case studies" da autora, Pamela Danese (2007); e, a baixa aplicação desse método no mercado também apresentado no artigo "Exploring the Impact of ICT in CPFR: A Case Study of an APS System in a Norwegian Pharmacy Supply Chain" dos autores, Maria Kollberg Thomassen, Heidi Dreyer e Patrik Jonsson (2012)";

5) Torna-se evidente por mais de um artigo selecionado que esses métodos devem ser seguidos por transformações estruturais, culturais, processuais e tecnológico do dia a dia das organizações. Um exemplo desse fato refere-se ao estudo de caso da Zara presente no artigo "The Study of Constructing Quick Response Mode in Fashion Industry" dos autores Mao, Zhu e Cui (2011) e do estudo de caso "Customer Service Logistics como Estratégia Organizacional: um estudo de caso em uma multinacional", de autoria de Da Silva (2018). Além disso, deve-se estudar a capacidade dos parceiros em responderem à colaboração de forma eficiente, eficaz e efetiva;

6) É evidente que mesmo sem os direcionamentos claros das ações realizadas nas empresas quanto aos métodos cooperativos, em vários artigos, as empresas conseguem ganhos reais e mensuráveis na diminuição de custos, aumento do giro de estoque e eficiência do gerenciamento da produção e logística;

7) Os métodos podem variar de acordo com a necessidade de frequência de entregas e volume de produtos. Por exemplo, o VMI deve conter um número menor de entregas e em maior volume, já o QR se especializa em maior frequência de entregas com um baixo volume;

8) A implementação dos métodos deve ser realizada de forma progressiva a fim de contornar resistências e se utilizar da aprendizagem organizacional, a qual detém uma curva de aprendizagem em " $U$ ". Além disso, a implementação do método pode ser referente a alguns parceiros da cadeia e de forma progressiva, bem como a grupos de produtos específicos que precisem de maior eficiência em sua operação para ganhar maior competitividade e valor agregado;

9) Por último, ressalta-se que as empresas podem utilizar da colaboração para ganhar benefícios unilaterais na cadeia, caso detenham maior poder que seus parceiros no processo. Problemas como este podem ser resolvidos com a utilização de taxas de redistribuição de lucros para todos os membros, fazendo com que toda a cadeia ganhe com a sua otimização de valor e dessa forma possam manter o negócio.

Embora tenha sido apresentado nesta seção, as principais técnicas e métodos e suas principais características, 0 amadurecimento das indústrias brasileiras quanto às práticas cooperativas ou colaborativas ainda se encontram em estágio inicial, ao considerar-se que a própria literatura ainda busca apresentar conceitos claros sobre 0 assunto. Diante disso, os resultados apresentados buscam através da contribuição teórica e revisão de literatura, uma definição clara sobre as técnicas e métodos, demonstrando quais são suas aplicações em estudos de casos e como os autores definiram em seus referidos trabalhos.

No entanto, na literatura ainda há poucos trabalhos a respeito da temática (GOMES; KLIEMANN NETO, 2015), e a disseminação dos métodos e práticas colaborativas ainda não implicaram em uma adesão, um dos motivos é a complexidade de suas implementações e a falta de educação formal e conhecimento dos profissionais dificultam sua implementação prática (GOMES; KLIEMANN NETO, 2015; DA SILVA, 2018).

Contudo, as estratégias organizacionais buscam implementar métodos de parcerias e cooperação interorganizacional, tendo por objetivo o compartilhamento de responsabilidades, compromissos e objetivos comuns, além de coordenação e sintonia de processo (BALLOU, 2009; DA SILVA; SILVA, 2018; VERSCHOORE; 
BALESTRIN, 2008; ABDALLAH; BARBIERI, 2014; GOMES; KLIEMANN NETO, 2015; DA SILVA, 2018), portanto essas práticas no Brasil ainda são embrionárias.

\section{CONSIDERAÇÕES FINAIS}

Este artigo buscou apresentar ferramentas utilizadas em práticas colaborativas na gestão da cadeia de suprimentos. Os resultados da pesquisa apontam uma análise descritiva quanto às ferramentas mais utilizadas, como: o Supply Chain Operations Reference-model (SCOR); o Quick Response (QR); o Continuos Replenishment Program (CRP); o Customer Service Logistics (CSL); o Vendor Management Inventory (VMI); 0 Efficient Consumer Response (ECR); e, o Collaborative Planning Forecasting Replenishment (CPFR).

Os dados da pesquisa apontam que a literatura ainda se encontra em um estado embrionário sobre os métodos cooperativos ou criação de redes empresariais, bem como, são generalistas. Isso demostra que a literatura ainda não traz insumos práticos e tangíveis em ações e resultados, como comparações numéricas de indicadores, como também implementação prática dos métodos apresentados neste trabalho, demonstrando suas implicações reais para as empresas. Assim, é perceptível que a falta de conceitos tangíveis, pode gerar a não aceitação prática dos métodos para as empresas, conduzindo-as a não buscarem a utilização do método, ou pior, a utilização não eficiente.

No entanto, como se trata de prática recente no Brasil, ainda são amplas as possibilidades de incremento e remodelamento desses métodos, além da probabilidade de criação de novas possibilidades. Espera-se que com o passar dos anos, os números de implementações desses métodos aumentem e estejam em um estado de maior consolidação a fim de que suas características sejam claramente evidentes.

Como análise dos resultados encontrados, destacamos algumas vantagens sobre a criação de redes de cooperação e métodos colaborativos: (a) diminuição de custos logísticos e de produção e estoques; (b) maior giro de estoques e diminuição destes; (c) eficiência na produção e no gerenciamento de estoques; (d) otimização dos lucros da cadeia; (e) maior nível de serviço; (f) capacidade de adaptação às demandas; (g) diminuição do "efeito chicote" na cadeia de suprimentos por meio de melhores previsões da demanda; (h) planejamento mais eficiente de produção; (i) aumento da vantagem competitiva; (j) trabalho mais cooperativo entre os integrantes da cadeia; e (k) maior eficácia na comunicação da cadeia.

Com base nos resultados anteriores, evidenciam-se análises e contribuições dessa pesquisa sobre os métodos colaborativos: (a) entendimento da necessidade de uma reestruturação das empresas quanto a sua estrutura e processos internos para que haja uma boa colaboração; (b) compreensão das curvas de aprendizado da empresa durante esse processo; e (c) diferenciação dos métodos colaborativos por meio da necessidade de volume de entrega de produtos, entre outros.

Referente a limitações desse trabalho destacam-se que as melhores práticas têm como origem outros países.

Para trabalhos futuros, sugere-se estudos detalhados dos métodos cooperativos em empresas de mercado, mostrando percepções dos profissionais que na prática gerenciam as cadeias de su primentos e tem a possibilidade de implementação dos métodos.

Sugere-se ainda, que os próximos estudos busquem evidências sobre as dificuldades de implementação dos métodos e como as organizações superaram este paradigma.

\section{REFERÊNCIAS}

ABDALA, E. C.; BARBIERI, J . C. Determinants of Sustainable Supply Chain: an Analysis of Mensuration Models of Pressures and Socio- Environmental Practices. J ournal of Operations and Supply Chain Management, $v$. 7, n. 2, p. 110-123, 2014.

ASRAWI, I.; SALEH, Y.; OTHMAN, M. Integrating drivers' differences in optimizing green supply chain management at tactical and operational levels. Computers and Industrial Engineering, v. 112, p. 122-134, 2017. 
ASHBY, A.; LEAT, M.; HUDSON-SMITH, M. Making connections: a review of supply chain management and sustainability literature. Supply Chain Management: An International Journal, v. 17, n. 5, p. 497-516, 2012.

BALESTRIN, A.; VERSCHORRE, J. R. Réplica - Redes são Redes ou Redes são Organizações?. Revista de Administração Contemporânea, RAC, Rio de Janeiro, v. 18, n. 4, p. 523-533, jul./ago. 2014. doi:http://dx.doi.org/10.1590/1982-849rac201410961.

BALLOU, R. H. Gerenciamento da cadeia de suprimentos / logística empresarial. 5. ed. Porto Alegre: Bookman, 2009.

BANOMYONG, R.; SUPATN, N. Selecting logistics providers in Thailand: a shippers' perspective. European Journal of Marketing, v. 45, n. 3, 2011.

BERTAGLIA, P. R. Logística e gerenciamento da cadeia de abastecimento. São Paulo: Saraiva, 2003.

BORGES, W. L. Uma análise na Implantação do CPFR em Empresas e Varejo. 2011. 168 f. Dissertação (Mestrado em Engenharia de Produção) - Universidade Federal de São Carlos, São Carlos, 2011.

CALATAYUD, A.; MANGAN, J .; CHRISTOPHER, M. The self-thinking supply chain. Supply Chain Management: An International J ournal, v. 24, n. 1, p.22-38, 2019.

CAO, M.; ZHANG, Q. Supply chain collaboration: impact on collaborative advantage and firm performance. Journal of Operations Management, v. 29, n. 3, p. 163-180. 2011.

CHEN, L. T. Dynamic supply coordination under consignment and vendor-managed inventory in retailercentric B2B electronic markets. Industrial Marketing

Management. v. 42, n. 4, p. 518-531, 2013.

CHOPRA, S.; SODHI, M. S. Managing risk to avoid supply chain breakdown. MIT Sloan Management Review. [S.I.], v. 46, n. 1, 2004.

CHRISTOPHER, M.; LEE, H. L. Mitigating supply chain risk through improved confidence. International J ournal of Physical Distribution \& Logistics Management, v. 34, n. 5, p. 388-396, 2004.

CHING, H, Y. Gestão de estoques na cadeia de logística integrada. 4. ed. São Paulo: Atlas, 2010.

CORRÊA, H. L.; CORRÊA, C. A. Administração de produção e operações: manufatura e serviços: uma abordagem estratégica. 2. ed. São Paulo: Atlas, 2007.

CRONIN, P.; RYAN, F.; COUGHLAN, M. Undertaking a literature review: a step-by-step approach. British Journal of Nursing, v. 17, n. 1, 2008.

CSCMP - COUNCIL OF SUPPLY CHAIN MANAGEMENT PROFESSIONALS. Supply Chain Operations Reference (SCOR $₫$ ) model. 2018. Disponível em:

http://www.portaldeconhecimentos.org.br/index.php/por/content/download/24758/296095/file/Supply. Acesso em: 04 mar. 2019.

DANESE, P. Designing CPFR collaborations: insights from seven case studies. International J ournal of Operations \& Production Management, v. 27, n. 2, p.181-204, 2007.

https://doi.org/10.1108/01443570710720612.

DAVIS, B. R.; MENTZER, J. T. Logistics Service Driven Loyalty: An Exploratory Study. Journal Of Business Logistics, v. 27, p. 53-73, maio 2011. https://doi.org/10.1002/j.2158-1592.2006.tb00217.x.

DA SILVA, J . A. B. Customer service logistics como estratégia organizacional: um estudo de caso em uma multinacional. Brazilian J ournal of Production Engineering, v. 4, n. 4, p. 226-244, 2018. 
DA SILVA, J. A. B.; SILVA, S. Critérios de qualidade em serviços de transporte público urbano: uma contribuição teórica. Brazilian Journal of Production Engineering, v. 4, n. 1, p. 83-98. 2018.

DA SILVA, J . A. B.; SCHLAG, F. Aspectos tangíveis e intangíveis na definição de qualidade do transporte público. In: SEMINÁRIO DE PESQUISA, PÓS-GRADUAÇÃO, ENSINO E EXTENSÃO DO CAMPUS ANÁPOLIS DE CSEH (SEPE), 3., 2017. Anais [...]. Anápolis: CSEH, 2017.

ELLRAM, L.; COOPER, M. Supply Chain Management: It's All About the J ourney, Not the Destination - Journal of Supply Chain Management, v. 50, n. 1, 2014.

GIL, A. C. Como elaborar projetos de pesquisa. 4. ed. São Paulo: Atlas, 2009.

GOMES, L. C.; KLIEMANN NETO, F.J. Métodos colaborativos na gestão de cadeias de sup rimentos: desafios de implementação. Revista de Administração de Empresas, v. 55, n. 5, p. 563-577, 2015.

http://dx.doi.org/10.1590/S0034-759020150508

GOMES, L. C.; KLIEMANN NETO, F. J. Métodos colaborativos na gestão de cadeias de suprimentos: desafios de implementação. Revista de administração de empresas. v.55, n. 5, p.563-577, 2015.

doi:http://dx.doi.org/10.1590/S0034-759020150508.

GUARNIERI, P. et al. Productive Agglomerations of Suppliers in the Automotive Industry: A Way to Maximize Competitiveness in Supply Chain Management. Journal of Technology Management \& Innovation, [S.I.], v. 1, n. 3, p. 38-46, Aug. 2006.

GHISI, F. A; SILVA, A. L. da. ECR entre o conceito e a prática: um estudo multicaso no canal de distribuição de mercearia básica. Organizações Rurais e Agroindustriais, Lavras, v. 7, n. 1, p. 37-49, 2005.

HASSINI, E.; SURTI, C.; SEARCY, C. A literature review and a case study of sustainable supply chains with a focus on metrics. International J ournal of Production Economics, v. 140, n. 1, p. 69-82, 2012.

LUO R. et al. A case study of suppy chain management in a Manufacturing Company in China. Nang Yan Business Journal, v. 6, n. 1, 2017.

HUDNURKAR, M.; JAKHAR, S.; RATHOD, U. Factors affecting collaboration in supply chain: a literature review. Procedia: Social and Behavioral Sciences,

v. 133, n. 15, p. 189-202, 2014. http://dx.doi.org/10.1016/j.sbspro.

LIU, H.et al. Effects of supply chain integration and market orientation on firm performance: Evidence from China. International J ournal of Operations \& Production Management, v. 33, n. 3, p. 322-346, 2013.

MAO, D.; ZHU, D.; CUI, Y. The study of constructing quick response mode in fashion industry. Fashion.Art Design Institute, Donghua University, Shanghai,China. Advanced Materials Research, v. 332-334, p. 359$362,2022$.

MATTHIAS, H; DISNEY, S; HOLMSTRON, J; SMAROS, J Supply Chain Collaboration: Making Sense of the Strategy Continuum. European Management Journal, v. 23, n. 2, p. 170-181, Apr. 2005.

MENTZER, J. T. et al. Defining Supply Chain Management. Journal of Business Logistics, v. 22, n. 2, 2001.

MORESI, E. A. D. Apostila de metodologia da pesquisa. Brasília: Universidade Católica de Brasília, 2003.

NAGALATHA, M; HUSSAIN, S. Potential of VMI Application in Commercial Vehicle Manufacturing IndustryA Case Study. International Journal of Research in Commerce \& Management, v. 2, n. 2, Feb. 2011.

NOVAES, A. G. Logística e gerenciamento da cadeia de distribuição. Rio de J aneiro: Elsevier, 2007. 
PIRES, S. R. I. Gestão da Cadeia de Suprimentos (Supply Chain Management) - Conceitos, Estratégias e Casos. São Paulo: Atlas, 2009.

PISSAIA, J. H.; CHAVES, C. A.; CARDOSO, Á. A. Análise estratégica na aplicabilidade do Vendor Managed Inventory (VMI) na cadeia de abastecimento. Universidade Nove de Julho. São Paulo: Revista Exacta, v. 10, n. 2, p. 207-222, 2012.

RAMANATHAN, U. Supply chain collaboration for improved forecast accuracy of promotional sales. International J ournal of Operations \& Production Management, v. 32, n. 6, p. 676-695, 2012. doi:10.1108/01443571211230925

RAMANATHAN, U.; GUNASEKARAN, A. Supply chain collaboration: Impact of success in long-term partnerships. Int. J. Production Economics, n. 147, p. 252-259, 2014.

SALZANO, W. Customer Service nas Atividades Logísticas. São Paulo: IMAM, 2008.

SAMPAIO, R. F; MANCINI, M. C. Estudo de Revisão Sistemática: Um Guia para Síntese Criteriosa da Evidência Científica. Revista Brasileira de Fisioterapia, São Carlos, v. 11, n. 1, p. 83-89, jan./fev. 2007.

SIMCH-LEVI, D; KAMINSKY, P.; SIMCHI-LEVI, E. Cadeia de Suprimentos: Projeto e Gestão. 3. ed. Porto Alegre: Bookman, 2010.

SUN, Y. Study on Manufacturing Industry Based on VMI. Lecture Notes in Electrical Engineering, London, v. 216, Springer, 2013. https://doi.org/10.1007/978-1-4471-4856-2_101

TONTINI, G.; ZANCHETT, R. Atributos de satisfação e lealdade em serviços logísticos. Gestão \& Produção, São Carlos, v.17, n. 4, out./dez. 2010. http://dx.doi.org/10.1590/S0104-530X2010000400013

THOMASSEN, M. K.; DREYER, H.; J ONSSON, P. Exploring the Impact of ICT in CPFR: A Case Study of an APS System in a Norwegian Pharmacy Supply Chain. IFIP Advances in Information and Communication Technology, Berlin, v. 398, Springer, 2012. https://doi.org/10.1007/978-3-642-40361-3.

VAN HOEK, R.; CHAPMAN, P. How to move supply chain beyond cleaning up after new product development. Supply Chain Management: An international Journal, v. 12 n. 4, p. 239 - 244, 2007.

VERSCHOORE, J. R.; BALESTRIN, A. Fatores relevantes para o estabelecimento de redes de cooperação entre empresas do Rio Grande do Sul. Revista de

Administração de Empresas, v. 12, n. 4, p. 043-1069, 2008.

WANKE, P. E. Logística para MBA em 12 lições. São Paulo: Atlas, 2010.

YAN, S. Empirical study on influencing factors of Quick Response ability for small and medium sized logistics enterprise. International Conference on Information Management, Innovation Management and Industrial Engineering IEEE Xplore, Xi'an, p. 1-4, 2013. doi: 10.1109/ICIII.2013.6703092

YIN, R. K. Estudo de Caso: planejamento e métodos. 5. ed. Porto Alegre: Bookman: 2015.

YAOA, Y. et al. Learning curves in collaborative planning, forecasting, and replenishment (CPFR) information systems: An empirical analysis from a mobile phone manufacturer. Journal of Operations Management, n. 31, p. 285-297, 2013

ZHANG, C. J. Study on Quick Response Mode of Agricultural Products Logistics under E-Commerce Environment. Applied Mechanics and Materials, v. 195-196, p. 1227-1231, 2012.

https://doi.org/10.4028/www.scientific.net/AMM.195-196.1227 
ZVIRGZDIN̦A, R; LININ̦A I; VĒVERE, V. Efficiente Consumer Response (ECR) Principles and theis Application in Retail Trade in Latvia. European Integration Studies, n. 9, p. 257-264, 2015. DOI:

http://dx.doi.org/10.5755/j01.eis.0.9.12812 